\title{
Surveillance des infections associées aux soins en réanimation : simplifier pour progresser?
}

\author{
Surveillance of Healthcare-associated Infection in the Intensive Care Unit: Simplifying \\ for Improvement
}

\author{
J.-C. Lucet \\ Reçu le 15 mai 2018; accepté le 18 mai 2018 \\ (C) SRLF et Lavoisier SAS 2018
}

Malgré les progrès réalisés ces dernières années, l'infection associée aux soins (IAS) acquise en réanimation reste la complication majeure dans ces unités.

Comme le rappelle Alain Lepape (p. 197), c'est la combinaison de deux facteurs qui explique la fréquence des IAS : d'abord, l'exposition aux dispositifs invasifs, portes d'entrée des bactéries responsables d'IAS et accessibles à la prévention ; ensuite, les facteurs endogènes que sont les comorbidités et l'immunodépression induite par le séjour en réanimation, eux non accessibles à la prévention.

\section{Bilan - contrasté — de l'acquis}

La surveillance nationale à travers le réseau Réa-Raisin regroupe un peu moins de la moitié des unités et des lits de réanimation en France. Les grandes tendances sur 12 ans de surveillance sont les suivantes [1] :

- une augmentation franche des scores de gravité, avec un score IGS2 à l'admission passant de 40 à 46 et un taux de mortalité en réanimation resté stable autour de $17 \%$, témoignant de l'amélioration de la prise en charge des patients ;

- une densité d'utilisation des procédures variables, de $50 \%$ pour l'intubation, à $2 / 3$ des journées pour la présence d'un cathéter veineux central et $80 \%$ des journées pour le sondage urinaire ;

- alors que le recours à l'intubation a augmenté durant la première période de surveillance, il semble plutôt en diminution actuellement, reflet peut-être d'un recours plus fréquent à la ventilation non invasive et à l'oxygénothérapie à haut débit ;

J.-C. Lucet $(\bowtie)$

UHLIN, GH Bichat-Claude-Bernard,

46, rue Henri Huchard, 75877 Paris cedex 18, Paris, France

e-mail : jean-christophe.lucet@bch.aphp.fr
- si le taux de bactériémies rapportées à 1000 jours/patient est resté stable, les infections liées au cathéter (ILC), malgré des taux initialement bas dès 2004, ont continué à diminuer d'environ $50 \%$ en dix ans, un succès à mettre sur le compte des stratégies de prévention généralisées dans les services (au moins ceux qui participent à la surveillance nationale) ;

- en revanche, le taux de pneumonies liées à l'intubation (PAVM) reste stable, aux alentours de 15 événements pour 1000 jours d'exposition, malgré la mise en œuvre des mesures de prévention recommandées.

D'autres évolutions sont à noter dans cette surveillance : d'abord, l'arrêt de la surveillance des infections urinaires, dont le diagnostic était souvent fait par défaut d'une autre infection documentée, en l'absence de critère clinique spécifique en situation de réanimation. La question est actuellement posée de l'intérêt de la culture systématique des cathéters veineux centraux, dont l'utilité est en effet discutable, au regard de la rareté des ILC.

Ces évolutions témoignent du souhait du comité de pilotage Réa-Raisin de restreindre la surveillance à la PAVM et à l'ILC : la PAVM, infection fréquente avec un impact sur le devenir du patient, mais d'évitabilité incertaine ; l'ILC vient presque en miroir de la PAVM, avec une infection maintenant rare, parce qu'accessible à la prévention.

Enfin, le réseau identifie les principales tendances de la résistance bactérienne. Ce sont les services de réanimation qui ont enregistré les premiers la réduction des taux d'infection à Staphylococcus aureus résistant à la méticilline (SARM), mais aussi ceux où la résistance des entérobactéries aux céphalosporines de troisième génération, dont les BLSE, a augmenté moins rapidement que dans les autres secteurs de soins hospitaliers.

Ces résultats montrent la mobilisation des services de réanimation pour la prévention des IAS. Faut-il alors continuer sur cette lancée sans prendre en compte les évolutions récentes du paysage du risque infectieux? 


\section{Quelles perspectives?}

Si la population des patients de réanimation est fortement exposée aux procédures invasives, le nombre de lits de réanimation, parmi l'ensemble des lits de court séjour, est faible, très inférieur à $5 \%$. Bien sûr, l'incidence des IAS est particulièrement élevée en réanimation, mais le nombre d'IAS est nettement supérieur en dehors des secteurs de réanimation.

\section{Cathéter central}

L'enquête nationale de prévalence des IAS de 2012 est éclairante : $42 \%$ des bactériémies associées aux soins étaient liées à une ILC, la prévalence des bactériémies associées au cathéter était cinq fois plus élevée en réanimation qu'en court séjour hors réanimation ( 3,2 vs $0,6 \%$ ), mais $84 \%$ des bactériémies associées au cathéter étaient observées en dehors de la réanimation [2].

La prévention des ILC en dehors de la réanimation y est plus complexe pour plusieurs raisons. La première est que l'expertise des équipes de soins hors réanimation n'y est pas la même qu'en réanimation, en dehors des secteurs spécialisés comme la nutrition parentérale, les hôpitaux de jour d'oncologie ou le secteur d'hémodialyse qui utilise des cathéters de Canaud. Quelques études comparant des pratiques de gestion des cathéters dans et hors réanimation montrent que la proportion de journées de cathéter non justifiées est nettement plus importante hors réanimation, et les pratiques de gestion des pansements y sont moins bonnes [3]. Par ailleurs, l'adhésion des pansements est moins bonne chez des patients devenus ambulatoires qu'elle ne l'était en réanimation pour des cathéters maintenant généralement posés par voie jugulaire.

Le Programme national de prévention 2015 des IAS (PROPIAS) a identifié dans son axe 3 les actions de surveillance et de prévention pour les actes invasifs : surveillance des ISO et prévention de l'ILC tout au long du parcours de soins, de la réanimation vers le MCO, les prises en charge ambulatoires et le domicile [4]. La réflexion d'un groupe de travail national en 2013 avait aussi identifié la surveillance et la prévention des ILC comme un champ encore peu ou pas investi en dehors des secteurs spécialisés.

À l'occasion de la régionalisation de la prévention des IAS avec la création des Cpias, le ministère de la Santé a demandé que les réseaux de surveillance, actuellement fédérés au sein du Raisin, soient redéfinis sous la coordination de Santé publique France. Le groupe de travail national a ainsi identifié cinq missions, l'une d'entre elles étant la surveillance et la prévention des infections associées aux actes invasifs dans l'ensemble du système de santé.

Le taux d'ILC en réanimation est maintenant extrêmement faible. Comment progresser en réanimation et en aval de la réanimation ? Une première réflexion est que la méthode de surveillance des ILC pourrait évoluer. Faut-il vraiment continuer à relever un dénominateur dans la surveillance des ILC en toutes circonstances, quand le nombre d'événements est de quelques unités par an et par service ? Il faut bien sûr continuer à produire des taux d'infection pour 1000 jours/cathéter, nécessaires pour la surveillance au niveau européen, et pour juger au niveau national de l'impact des efforts de prévention. En revanche peut-être faudrait-il aussi y associer une approche individuelle, où chaque ILC ferait l'objet d'une analyse des causes, pour identifier d'éventuels défauts de prévention.

Une deuxième voie pour progresser sur la prévention et intégrer le séjour en réanimation avec l'aval de réanimation est de s'intéresser à la présence du cathéter veineux central à la sortie de réanimation. Les données Réa-Raisin indiquent qu'environ un tiers des cathéters, le plus souvent CVC de courte durée, restent en place à la sortie des patients vers le secteur d'aval [1]. Beaucoup de ces CVC restent nécessaires pour l'administration de médicaments ou une nutrition parentérale ; une mesure de prévention simple est de retirer tout cathéter inutile à la sortie des patients et, pour ceux dont le cathéter reste nécessaire, d'envisager la pose d'un PICC, dont la gestion du pansement est plus simple et moins à risque que le cathéter jugulaire chez un patient autonome.

Il est aussi probable que l'ouverture de la surveillance et celle de la prévention à l'ensemble des cathéters centraux et périphériques dans le système de santé seront une occasion pour améliorer les pratiques de prévention en dehors de la réanimation $[5,6]$. En somme, il s'agit de transposer l'expertise acquise en réanimation vers les autres secteurs de soins, avec la difficulté de s'adresser à un beaucoup plus grand nombre de soignants plus rarement confrontés à la gestion d'un cathéter veineux central.

\section{Pneumonie sous ventilation mécanique}

La prévention et la surveillance des PAVM posent des questions différentes. Force est de constater que les taux d'infection n'ont pas changé en France depuis 15 ans malgré les efforts de prévention, avec des taux rapportés notablement plus élevés que dans d'autres surveillances nationales, comme en Allemagne ou aux États-Unis. Les raisons de ces différences majeures de taux de PAVM sont multiples.

Il peut bien sûr s'agir de différences dans la qualité des mesures de prévention. Mais la comparaison des taux d'ILC en France et dans d'autres pays montre que la France n'a rien à envier aux autres pays, bien au contraire, et les explications sont à chercher en partie ailleurs.

Aux États-Unis, les infections associées à la ventilation mécanique ne sont plus remboursées dans le système Medicare et Medicaid, si bien que les réanimateurs américains n'ont plus de raison de les rapporter [7] ; c'est d'autant 
plus vrai que ces données font l'objet d'une diffusion publique dans la majorité des états américains, d'ailleurs sous des présentations différentes, du nombre brut de PAVM jusqu'au taux d'incidence pour 1000 jours de ventilation mécanique [8].

Un deuxième facteur est que la définition des PAVM est la plus complexe parmi les IAS, qu'il s'agisse des critères cliniques, radiologiques, biologiques ou microbiologiques. La pratique de prélèvements microbiologiques distaux est communément acceptée en France, mais les autres critères diagnostiques sont variables selon l'œil et l'inquiétude du réanimateur. Il n'est pas certain que l'arrivée des VAE (ventilatorassociated events), parmi lesquels les pneumonies possibles ou probables, permette de régler ces difficultés diagnostiques.

On peut aussi se demander si les critères diagnostiques français, souvent fondés sur des prélèvements distaux protégés, conduisent à prendre en compte un compte bactériologique significatif pour faire le diagnostic de PAVM et paradoxalement augmentent artificiellement le taux de PAVM si la décision thérapeutique n'est fondée que sur le compte bactérien.

Il n'empêche que, puisque les taux de PAVM n'ont pas diminué chez les patients ventilés, la meilleure mesure de prévention est de ne pas intuber les patients. Sur le plan diagnostique, peut-être faudrait-il aussi intégrer un critère qui serait le traitement d'une infection d'origine pulmonaire pendant une durée minimale, celle-ci correspondant alors probablement à la prise en charge d'une infection pulmonaire, quel qu'en soit le siège : trachéobronchite ou pneumonie.

\section{Surveillance et/ou prévention?}

Le dogme établi par le Senic Project des CDC aux États-Unis dans les années 1970 a été de fonder la politique de prévention sur l'obtention de données de taux d'infection que les équipes de soins et de prévention d'infections peuvent utiliser pour la réflexion et les actions de prévention [9]. Ce paradigme évolue vers des actions de prévention associant des indicateurs de résultats - les taux d'infection — et des indicateurs de bonnes pratiques, sous l'impulsion des travaux s'intéressant aux bundles de prévention. Ainsi, le réseau européen de surveillance en réanimation animé par l'ECDC propose d'associer à la surveillance des IAS des indicateurs de bonnes pratiques de prévention autour de l'hygiène des mains, du ratio de personnel, de la prévention des ILC, des PAVM et des bonnes pratiques d'antibiothérapie.

Cette évolution associant surveillance et prévention des IAS permet aux équipes de prévention et de contrôle de l'infection d'être plus présentes dans les services de réanimation, et pas uniquement pour faire la surveillance, mais pour participer à la prévention autour des soins.

\section{Quelle surveillance?}

Une dernière perspective est celle des modalités de surveillance. Les différentes méthodes sont revues par C. Bourigault et al. dans ce même numéro (p. 204). Il est surprenant et inquiétant qu'en 2018 la majorité des services effectuant la surveillance continuent à le faire à partir d'un document individuel ouvert pour chaque patient, souvent en format papier. Il est urgent que nos systèmes informatiques permettent de récupérer, de façon quasi automatique, le dénominateur, durées de ventilation et de cathéter.

La surveillance particulière des IAS en réanimation patient-based, particulière à la France, permet de générer des données individuelles très utiles pour mieux détailler le risque infectieux. Faut-il continuer à faire une surveillance patient-based ou plutôt unit-based, plus simple, comme réalisée actuellement dans les réseaux allemands et étatsuniens ? C'est une réflexion à mener pour simplifier le recueil de données en attendant que les supports informatiques soient disponibles dans toutes les unités.

\section{Conclusion}

Notre système de soins évolue avec, en court séjour, un recours plus fréquent à un accès veineux central, jusqu'alors l'apanage des services de réanimation. Il est donc nécessaire de transposer les bonnes pratiques établies en réanimation vers les autres secteurs de soins. C'est l'enjeu de la redéfinition des missions nationales de surveillance et de prévention, actuellement en construction.

Quant aux PAVM, si leur surveillance et leur prévention restent difficiles, elles participent pour plus de la moitié des antibiothérapies débutées en réanimation. Malgré les efforts de prévention, leurs taux stagnent. Il serait utile de réfléchir collectivement aux évolutions possibles. La publication prochaine des résultats de l'étude européenne R-Gnosis en réanimation nous dira si la décontamination digestive sélective est une piste, malgré les risques d'impact sur l'évolution des résistances inhérents à cette pratique.

De nouveaux risques apparaissent, par exemple ceux associés à l'ECMO : le niveau de risque et les mesures de prévention n'en sont pas encore bien définis.

Enfin, la question des bonnes pratiques d'antibiothérapie n'est pas discutée ici, mais serait certainement un autre aspect des bonnes pratiques à aborder, moins sur la durée d'antibiothérapie qui semble maintenant réduite aux durées courtes recommandées pour les PAVM que pour les décisions d'initiation ou de réévaluation des antibiotiques au vu des résultats bactériologiques. 


\section{Références}

1. Réseau d'alerte d'investigation et de surveillance des infections nosocomiales (Raisin), (2017) Surveillance des infections nosocomiales en réanimation adulte, réseau Réa-Raisin, France, Résultats 2015. [15 janvier 2018]. Available from: http://www. invs.sante.fr/\%20fr/Dossiers-thematiques/Maladies-infectieuses/ Infections-associees-aux-soins/Surveillance-des-infections-associeesaux-soins-IAS

2. Giard M, Machut A, Noel-Lagnado D, Ayzac L, Vaux S, Coignard B, Savey A; pour le groupe de travail ENP-Raisin 2012, (2014) Analyse comparative des bactériémies nosocomiales chez l'adulte en réanimation et hors réanimation : enquête de prévalence 2012, France. Bull Epidemiol Hebdo 29: 474-481

3. Trick WE, Vernon MO, Welbel SF, Wisniewski MF, Jernigan JA, Weinstein RA, (2004) Unnecessary use of central venous catheters: the need to look outside the intensive care unit. Infect Control Hosp Epidemiol 25: 266-268

4. Ministère des Affaires sociales dlsedddf, (2015) Programme national d'actions de prévention des infections associées aux soins
(Propias) 2015. Available from: http://solidarites-sante.gouv.fr/ IMG/pdf/2015_202to.pdf

5. Zingg W, Cartier V, Inan C, Touveneau S, Theriault M, GayetAgeron A, Clergue F, Pittet D, Walder B, (2014) Hospital-wide multidisciplinary, multimodal intervention programme to reduce central venous catheter-associated bloodstream infection. PLoS One 9: e93898 (Epub 2014/04/10)

6. Freixas N, Bella F, Limon E, Pujol M, Almirante B, Gudiol F, (2013) Impact of a multimodal intervention to reduce bloodstream infections related to vascular catheters in non-ICU wards: a multicentre study. Clin Microbiol Infect 19: 838-844 (Epub 2012/11/08)

7. Pronovost PJ, Goeschel CA, Wachter RM, (2008) The wisdom and justice of not paying for "preventable complications". Jama 299: 2197-2199 (Epub 2008/05/15)

8. Haut ER, Pronovost PJ, (2011) Surveillance bias in outcomes reporting. Jama 305: 2462-2463 (Epub 2011/06/16)

9. Haley RW, Culver DH, White JW, Morgan WM, Emori TG, Munn VP, Hooton TM, (1985) The efficacy of infection surveillance and control programs in preventing nosocomial infections in US hospitals. Am J Epidemiol 121: 182-205 